\title{
SOLUTIONS FOR NONLINEAR VARIATIONAL INEQUALITIES WITH A NONSMOOTH POTENTIAL
}

\author{
MICHAEL E. FILIPPAKIS AND NIKOLAOS S. PAPAGEORGIOU
}

Received 4 November 2003

First we examine a resonant variational inequality driven by the $p$-Laplacian and with a nonsmooth potential. We prove the existence of a nontrivial solution. Then we use this existence theorem to obtain nontrivial positive solutions for a class of resonant elliptic equations involving the $p$-Laplacian and a nonsmooth potential. Our approach is variational based on the nonsmooth critical point theory for functionals of the form $\varphi=\varphi_{1}+\varphi_{2}$ with $\varphi_{1}$ locally Lipschitz and $\varphi_{2}$ proper, convex, lower semicontinuous.

\section{Introduction}

In this paper, we consider the following nonlinear variational inequality at resonance with a nonsmooth potential function $\left(Z \subseteq R^{N}\right.$ is a bounded domain with a $C^{2}$-boundary $\left.\partial Z\right)$ :

$$
\begin{gathered}
\int_{Z}\|D x(z)\|^{p-2}(D x(z), D y(z)-D x(z))_{R^{N}} d z-\lambda_{1} \int_{Z}|x(z)|^{p-2} x(z)(y-x)(z) d z \\
\geq \int_{Z} u(z)(y-x)(z) d z \quad \forall y \in C,
\end{gathered}
$$

where $C=\left\{x \in W_{0}^{1, p}(Z): x(z) \geq g(z)\right.$ a.e on $\left.Z\right\}$, with $g \in W^{1, p}(Z), g(z) \leq 0$ a.e. on $Z$, and $u \in L^{q}(Z),(1 / p+1 / q=1,1<p<\infty), u(z) \in \partial j(z, x(z))$ a.e. on $Z$. Here, the potential function $z \rightarrow j(z, x)$ is only locally Lipschitz, not necessarily $C^{1}$, and $\partial j(z, \cdot)$ denotes the generalized (Clarke) subdifferential (see Section 2). In addition, $\lambda_{1}>0$ stands for the first eigenvalue of the negative $p$-Laplacian with Dirichlet boundary condition (denoted by $\left.\left(-\triangle_{p}, W_{0}^{1, p}(Z)\right)\right)$.

Also, we study the following nonlinear elliptic problem at resonance with nonsmooth potential:

$$
\begin{gathered}
-\operatorname{div}\left(\|D x(z)\|^{p-2} D x(z)\right)-\lambda_{1}|x(z)|^{p-2} x(z) \in \partial j(z, x(z)) \quad \text { a.e on } Z, \\
\left.x\right|_{\partial Z}=0, \quad 1<p<\infty, x \geq 0 .
\end{gathered}
$$


Having as a starting point a solution of problem (1.1) when $g \equiv 0$, we show that problem (1.2) has at least one nontrivial positive solution. Moreover, by strengthening a little our hypotheses, we show that the solution is in fact strictly positive and smooth.

Problems similar to (1.1) were investigated by Szulkin [19] and more recently by Le $[13,14]$ and Zhou and Huang [22]. In [19], the problem under consideration is semilinear (i.e., $p=2$ ), not in resonance, $g \equiv 0$, and the potential function is smooth (i.e., a $C^{1}$ function). His approach is variational. Le $[13,14]$ considers nonlinear variational inequalities not in resonance, which though, are driven by general nonlinear differential operators, which include as a special case the $p$-Laplacian. The right-hand side nonlinearity is Carathéodory (thus the corresponding potential function is $C^{1}$ ) and it may also depend on the gradient of the unknown function (see Le [13]). His approach is based on the method of upper and lower solutions. Moreover, we mention that in Le [14] the interested reader can find a rich bibliography on the subject of variational inequalities. In Zhou and Huang [22], the problem is nonlinear, not in resonance, with smooth potential. The approach in that paper is based on the theory of nonlinear complementarity problems.

Problems like (1.2) were studied in the context of semilinear equations (i.e., $p=2$ ) which are either nonresonant (see Alves and Miyagaki [1], Zhou [21]) or are near-resonance (see Mawhin and Schmitt [16] (ordinary differential equations) and Chiappinelli et al. [3] (elliptic equations)). For the near resonance problems, the authors obtain multiplicity results. For nonlinear problems driven by the $p$-Laplacian, the investigation is lagging behind and only recently Kyritsi and Papageorgiou [11] extended the aforementioned works on nearly resonant problems.

Unilateral problems with nonsmooth potential are also known in the literature as "variational-hemivariational inequalities" and arise in mechanics and engineering when one wants to consider more realistic nonmonotone and multivalued laws. We refer to the book of Naniewicz and Panagiotopoulos [17] and the paper of Goeleven et al. [7]. We should mention that our work here generalizes in many respects the semilinear eigenvalue problems studied by Goeleven et al. [7].

Our approach is variational, based on the nonsmooth extension of the theory of Szulkin [19], due to Kourogenis et al. [9]. For the convenience of the reader, in the next section we recall the basic definitions and facts from this theory, as well as relevant notions from convex analysis and the subdifferential theory for locally Lipschitz functions. For details, we refer to the books of Clarke [4] and Denkowski et al. [6].

\section{Mathematical background}

Let $X$ be a Banach space and $X^{*}$ its topological dual. By $\langle\cdot, \cdot\rangle$ we denote the duality brackets for the pair $\left(X, X^{*}\right)$. A function $\varphi: X \rightarrow \mathbb{R}$ is said to be locally Lipschitz, if for all $x \in X$, we can find a neighborhood $U$ of $x$ and a constant $k_{U}>0$ such that $|\varphi(y)-\varphi(u)| \leq$ $k_{U}\|y-u\|$, for all $y, u \in U$. From convex analysis, we know that if $\psi: X \rightarrow \overline{\mathbb{R}}=\mathbb{R} \cup\{+\infty\}$ is convex, lower semicontinuous, and proper (i.e., $\psi$ is not identically $+\infty$ ), then $\psi$ is locally Lipchitz in the interior of its effective domain $\operatorname{dom} \psi=\{x \in X: \psi(x)<+\infty\}$. In particular, then a continuous, convex function $\psi: X \rightarrow \mathbb{R}$ is locally Lipschitz. In analogy to the directional derivative of a convex function, for a locally Lipschitz function $\varphi: X \rightarrow R$, 
we define the generalized directional derivative of $\varphi$ at $x$ in the direction $h \in X$ by

$$
\varphi^{0}(x ; h)=\limsup _{\substack{x^{\prime} \rightarrow x \\ \lambda \downarrow 0}} \frac{\varphi\left(x^{\prime}+\lambda h\right)-\varphi\left(x^{\prime}\right)}{\lambda} .
$$

It is easy to check that $\varphi^{0}(x ; \cdot)$ is sublinear continuous, so it is the support function of a nonempty, $w^{*}$-compact, and convex set $\partial \varphi(x)=\left\{x^{*} \in X^{*}:\left\langle x^{*}, h\right\rangle \leq \varphi^{0}(x ; h)\right.$ for all $h \in$ $X\}$. The multifunction $\partial \varphi: X \rightarrow 2^{X^{*}} \backslash\{\varnothing\}$ is called the generalized (or Clarke) subdifferential of $\varphi$. If $\varphi$ is also convex, then the generalized subdifferential coincides with the subdifferential in the sense of convex analysis, given by $\partial_{c} \varphi(x)=\left\{x^{*} \in X^{*}:\left\langle x^{*}, y-x\right\rangle \leq\right.$ $\varphi(y)-\varphi(x)$ for all $y \in X\}$. Moreover, if $\varphi \in C^{1}(X)$, then $\partial \varphi(x)=\left\{\varphi^{\prime}(x)\right\}$. If $\varphi, \psi: X \rightarrow \mathbb{R}$ are locally Lipschitz functions and $\lambda \in R$, then for all $x \in X$, we have

$$
\partial(\varphi+\psi)(x) \subseteq \partial \varphi(x)+\partial \psi(x), \quad \partial(\lambda \varphi)(x)=\lambda \partial \varphi(x)
$$

Let $\Gamma_{0}(X)$ be the cone of convex, lower semicontinuous, proper functions. Let $\varphi_{1}$ : $X \rightarrow \mathbb{R}$ be locally Lipschitz, $\varphi_{2} \in \Gamma_{0}(X)$, and set $\varphi=\varphi_{1}+\varphi_{2}$. For such functions exists a nonsmooth critical point theory (see Kourogenis et al. [9]), which extends the theory of Szulkin [19], where $\varphi_{1} \in C^{1}(X)$. We say that $x \in X$ is a critical point of $\varphi=\varphi_{1}+\varphi_{2}$ if

$$
\varphi_{1}^{0}(x ; y-x)+\varphi_{2}(y)-\varphi_{2}(x) \geq 0 \quad \forall y \in X .
$$

We say that $\varphi=\varphi_{1}+\varphi_{2}$ satisfies the generalized nonsmooth Palais-Smale condition (generalized nonsmooth PS-condition for short), if every sequence $\left\{x_{n}\right\}_{n \geq 1} \subseteq X$ such that $\left\{\varphi\left(x_{n}\right)\right\}_{n \geq 1}$ is bounded and there exists $\left\{\varepsilon_{n}\right\}_{n \geq 1} \subseteq R_{+}, \varepsilon_{n} \downarrow 0$ such that

$$
\varphi_{1}^{0}\left(x_{n} ; y-x_{n}\right)+\varphi_{2}(y)-\varphi_{2}\left(x_{n}\right) \geq-\varepsilon_{n}\left\|y-x_{n}\right\| \quad \forall y \in X,
$$

has a strongly convergent subsequence. Remark that if $\varphi_{2} \equiv 0$, then using Szulkin [19, Lemma 3], we can find $x_{n}^{*} \in X^{*},\left\|x_{n}^{*}\right\| \leq 1$, such that

$$
\begin{aligned}
\varphi_{1}^{0}\left(x_{n} ; h\right) & \geq \varepsilon_{n}\left\langle x_{n}^{*}, h\right\rangle \quad \forall h \in X, \\
& \Longrightarrow \varepsilon_{n} x_{n}^{*} \in \partial \varphi_{1}\left(x_{n}\right) \quad \forall n \geq 1 .
\end{aligned}
$$

So it follows that $m\left(x_{n}\right)=\inf \left[\left\|u^{*}\right\|: u^{*} \in \partial \varphi_{1}\left(x_{n}\right)\right] \leq \varepsilon_{n} \rightarrow 0$ as $n \rightarrow \infty$ and we recover the nonsmooth PS-condition introduced by Chang [2] (see also Kourogenis and Papageorgiou [10] for extensions).

Moreover, if $\varphi_{1} \in C^{1}(X)$ and $\varphi_{2} \equiv 0$, we recover the classical PS-condition (see Denkowski et al. [5, page 171] and Struwe [18, page 70]). The following generalized nonsmooth mountain pass theorem can be found in Kourogenis et al. [9].

Theorem 2.1. If $X$ is a Banach space, $\varphi: X \rightarrow \overline{\mathbb{R}}=\mathbb{R} \cup\{+\infty\}, \varphi=\varphi_{1}+\varphi_{2}$ with $\varphi_{1}: X \rightarrow \mathbb{R}$ locally Lipschitz and $\varphi_{2} \in \Gamma_{0}(X), \varphi$ satisfies the generalized nonsmooth PS-condition, and there exist $r>0$ and $x_{0} \in X$ with $\left\|x_{0}\right\|>r$ such that

$$
\inf [\varphi(x):\|x\|=r] \geq \beta>\max \left\{\varphi(0), \varphi\left(x_{0}\right)\right\}
$$


then $\varphi$ has a critical point $x \in X$ with corresponding critical value $c=\varphi(x)$ given by

$$
\left.c=\inf _{\gamma \in \Gamma \in[0,1]} \max _{t \in[} \varphi(t)\right),
$$

where $\Gamma=\left\{\gamma \in C([0,1], X): \gamma(0)=0, \gamma(1)=x_{0}\right\}$. Moreover, $c \geq \beta$.

Since our problems are at resonance, they involve the principal eigenvalue $\lambda_{1}$ of $\left(-\triangle_{p}\right.$, $\left.W_{0}^{1, p}(Z)\right)$. So, very briefly, we recall what is known about $\lambda_{1}$. For details, we refer to Denkowski et al. [5]. So, on $Z$, we consider the following nonlinear eigenvalue problem:

$$
\begin{gathered}
-\operatorname{div}\left(\|D x(z)\|^{p-2} D x(z)\right)=\lambda|x(z)|^{p-2} x(z) \quad \text { a.e on } Z, \\
\left.x\right|_{\partial Z}=0, \quad 1<p<\infty, \quad \lambda \in R .
\end{gathered}
$$

Every $\lambda \in R$ for which (2.8) has a nontrivial solution is said to be an eigenvalue of $\left(-\triangle_{p}, W_{0}^{1, p}(Z)\right)$ and the nontrivial solution $x \in W_{0}^{1, p}(Z)$ is a corresponding eigenfunction. Using as a test function $x \in W_{0}^{1, p}(Z)$, we see that every eigenvalue $\lambda$ is nonnegative. In fact, the first eigenvalue $\lambda_{1}$ is strictly positive, isolated, and simple (i.e., the corresponding eigenspace is one-dimensional). Moreover, there is a variational characterization of $\lambda_{1}>0$ via a Rayleigh quotient,

$$
\lambda_{1}=\inf \left[\frac{\|D x\|_{p}^{p}}{\|x\|_{p}^{p}}: x \in W_{0}^{1, p}(Z), x \neq 0\right] .
$$

This infimum is realized at the corresponding normalized eigenfunction $u_{1}$. Note that in (2.9), we can replace $x$ by $|x|$, and so we infer that $u_{1}$ does not change sign on $Z$. Moreover, from nonlinear regularity theory, we know that $u_{1} \in C^{1}(\bar{Z})$ and we can say that $u_{1}(z)>0$ for all $z \in Z$.

\section{Generalized variational inequalities}

In this section, we deal with problem (1.1). Our hypotheses on the nonsmooth potential $j(z, x)$ are the following:

$\mathrm{H}(\mathrm{j}) \mathrm{j}: Z \times \mathbb{R} \rightarrow \mathbb{R}$ is a function such that $j(z, 0)=0$ a.e. on $Z$ and

(i) for all $x \in \mathbb{R}, z \rightarrow j(z, x)$ is measurable;

(ii) for almost all $z \in Z, x \rightarrow j(z, x)$ is locally Lipschitz;

(iii) for every $M>0$, there exists $\alpha_{M} \in L^{\infty}(Z)$ such that for almost all $z \in Z$, all $|x| \leq M$, and all $u \in \partial j(z, x)$, we have $|u| \leq \alpha_{M}(z) ;$

(iv) $\lim _{x \rightarrow+\infty}\left(u / x^{p-1}\right)=0$ uniformly for almost all $z \in Z$ and all $u \in \partial j(z, x)$;

(v) let $g_{1}(z, x)=\max [u: u \in \partial j(z, x)], g_{0}(z, x)=\min [u: u \in \partial j(z, x)]$, and

$$
G_{i}(z, x)=\left\{\begin{array}{ll}
\frac{p j(z, x)}{x}-g_{i}(z, x) & \text { if } x \neq 0, \\
0 & \text { if } x=0,
\end{array} \quad i=0,1 .\right.
$$


We assume that there exists a function $G_{+} \in L^{1}(Z)$ such that $G_{+}(z)=$ $\liminf _{x \rightarrow+\infty} G_{1}(z, x)$ uniformly for almost all $z \in Z, \int_{Z} G_{+}(z) u_{1}(z) d z>0$, and $G_{0}(z, x) \leq \theta(z), \theta \in L^{1}(Z) ;$

(vi) $\limsup _{x \rightarrow 0^{+}}\left(p j(z, x) / x^{p}\right)<0$ uniformly for almost all $z \in Z$.

Remark 3.1. Hypothesis $\mathrm{H}(\mathrm{j})(\mathrm{v})$ is an extension of the classical Landesman-Lazer condition.

Let $\varphi_{1}: W_{0}^{1, p}(Z) \rightarrow \mathbb{R}$ be the function defined by

$$
\varphi_{1}(x)=\frac{1}{p}\|D x\|_{p}^{p}-\frac{\lambda_{1}}{p}\|x\|_{p}^{p}-\int_{Z} j(z, x(z)) d z .
$$

We know that $\varphi_{1}$ is locally Lipschitz (see $\mathrm{Hu}$ and Papageorgiou [8, page 313]). Also, we set

$$
\varphi_{2}(x)=i_{C}(x)= \begin{cases}0 & \text { if } x \in C, \\ \infty & \text { if } x \notin C .\end{cases}
$$

Since $C \subseteq W_{0}^{1, p}(Z)$ is nonempty, closed, and convex, we have that $i_{C} \in \Gamma_{0}\left(W_{0}^{1, p}(Z)\right)$. We define

$$
\varphi=\varphi_{1}+\varphi_{2}
$$

Proposition 3.2. If hypotheses $H(j)$ hold, then $\varphi$ satisfies the generalized nonsmooth PScondition.

Proof. Let $\left\{x_{n}\right\}_{n \geq 1} \subseteq W_{0}^{1, p}(Z)$ be a sequence such that $\left|\varphi\left(x_{n}\right)\right| \leq M_{1}$ for some $M_{1}>0$, all $n \geq 1$, and $\varphi_{1}^{0}\left(x_{n} ; y-x_{n}\right)+\varphi_{2}(y)-\varphi_{2}\left(x_{n}\right) \geq-\varepsilon_{n}\left\|y-x_{n}\right\|$ for all $y \in W_{0}^{1, p}(Z)$.

Evidently, $\left\{x_{n}\right\}_{n \geq 1} \subseteq C$. We can find $x_{n}^{*} \in \partial \varphi_{1}\left(x_{n}\right)$ such that $\varphi_{1}^{0}\left(x_{n} ; y-x_{n}\right)=\left\langle x_{n}^{*}, y-\right.$ $\left.x_{n}\right\rangle$. This is a consequence of the fact that $\varphi_{1}^{0}\left(x_{n} ; \cdot\right)$ is the support function of $\partial \varphi_{1}\left(x_{n}\right)$ and the latter is $w$-compact in $W^{-1, q}(Z)=W_{0}^{1, p}(Z)^{*}$. For every $n \geq 1$, we have

$$
x_{n}^{*}=A\left(x_{n}\right)-\lambda_{1}\left|x_{n}\right|^{p-2} x_{n}-u_{n}
$$

Here, $A: W_{0}^{1, p}(Z) \rightarrow W^{-1, q}(Z)$ is the nonlinear operator defined by

$$
\langle A(x), w\rangle=\int_{Z}\|D x(z)\|^{p-2}(D x(z), D w(z))_{R^{N}} d z, \quad \forall x, w \in W_{0}^{1, p}(Z),
$$

and $u_{n} \in L^{q}(Z), u_{n}(z) \in \partial j\left(z, x_{n}(z)\right)$ a.e. on $Z$ (see Clarke [4, page 83] and Denkowski et al. [6, page 617]). It is easy to check that $A$ is monotone, demicontinuous, hence it is maximal monotone. 
We claim that $\left\{x_{n}\right\}_{n \geq 1} \subseteq W_{0}^{1, p}(Z)$ is bounded. Suppose that this is not the case. By passing to a suitable subsequence if necessary, we may assume that $\left\|x_{n}\right\| \rightarrow \infty$. Set $v_{n}=$ $x_{n} /\left\|x_{n}\right\|, n \geq 1$. Evidently, $\left\|v_{n}\right\|=1, n \geq 1$, and so we may assume that

(i) $v_{n} \stackrel{w}{\rightarrow} v$ in $W_{0}^{1, p}(Z), v_{n} \rightarrow v$ in $L^{p}(Z)$ (from the compact embedding of $W_{0}^{1, p}(Z)$ into $\left.L^{p}(Z)\right)$,

(ii) $v_{n}(z) \leq v(z)$ a.e on $Z$ and $\left|v_{n}(z)\right| \leq k(z)$ a.e on $Z, n \geq 1$, with $k \in L^{p}(Z)$.

(See Denkowski et al. [6, page 147]).

By virtue of hypotheses $\mathrm{H}(\mathrm{j})$ (iii) and (iv), given $\varepsilon>0$, we can find $\alpha_{\varepsilon} \in L^{\infty}(Z)$ such that for almost all $z \in Z$, all $x \geq 0$, and all $u \in \partial j(z, x)$, we have

$$
|u| \leq \alpha_{\varepsilon}(z)+\varepsilon x^{p-1}
$$

Using the mean value theorem for locally Lipschitz functions (see Clarke [4, page 41] and Denkowski et al. [6, page 609]), for almost all $z \in Z$ and all $x \geq 0$, we have

$$
\begin{aligned}
& \left.|j(z, x)| \leq|j(z, 0)|+\alpha_{\varepsilon}(z)|x|+\varepsilon|x|^{p} \leq \beta_{\varepsilon}(z)+2 \varepsilon|x|^{p} \quad \text { (with } \beta_{\varepsilon} \in L^{1}(Z)\right) \\
& \Longrightarrow \limsup _{n \rightarrow \infty}\left|\int_{z} \frac{j\left(z, x_{n}(z)\right)}{\left\|x_{n}\right\|^{p}} d z\right| \leq 2 \varepsilon
\end{aligned}
$$

$(\operatorname{see}(3.7))$.

Let $\varepsilon \downarrow 0$ to conclude that $\int_{Z}\left(j\left(z, x_{n}(z)\right) /\left\|x_{n}\right\|^{p}\right) d z \rightarrow 0$ as $n \rightarrow \infty$. From the choice of the sequence $\left\{x_{n}\right\}_{n \geq 1} \subseteq W_{0}^{1, p}(Z)$, we have

$$
\frac{\varphi\left(x_{n}\right)}{\left\|x_{n}\right\|^{p}}=\frac{1}{p}\left\|D v_{n}\right\|_{p}^{p}-\frac{\lambda_{1}}{p}\left\|v_{n}\right\|_{p}^{p}-\int_{Z} \frac{j\left(z, x_{n}(z)\right)}{\left\|x_{n}\right\|^{p}} d z \leq \frac{M_{1}}{\left\|x_{n}\right\|^{p}}
$$

(since $\varphi_{2}\left(x_{n}\right)=0$, because $x_{n} \in C$ ).

Passing to the limit as $n \rightarrow \infty$ and using the weak lower semicontinuity of the norm functional, we obtain

$$
\begin{aligned}
& \frac{1}{p}\|D v\|_{p}^{p} \leq \frac{\lambda_{1}}{p}\|v\|_{p}^{p} \\
& \Longrightarrow \frac{1}{p}\|D v\|_{p}^{p}=\frac{\lambda_{1}}{p}\|v\|_{p}^{p} \\
& \Longrightarrow v= \pm u_{1} \quad \text { or } \quad v=0
\end{aligned}
$$

(see [4]).

If $v=0$, then $\left\|D v_{n}\right\|_{p} \rightarrow 0$, and so $v_{n} \rightarrow 0$ in $W_{0}^{1, p}(Z)$, a contradiction to the fact that $\left\|v_{n}\right\|=1$ for all $n \geq 1$. So, $v= \pm u_{1}$. Recall that $x_{n}(z) \geq g(z)$ a.e. on $Z, n \geq 1$. Hence, $v_{n}(z) \geq g(z) /\left\|x_{n}\right\|$ a.e. on $Z$, and so, in the limit, we have $v(z) \geq 0$ a.e on $Z$. Therefore, $v=u_{1}$.

Now we fix $y=0 \in C$. From the choice of the sequence $\left\{x_{n}\right\}_{n \geq 1} \subseteq C$, we have

$$
\left\|D x_{n}\right\|_{p}^{p}-\lambda_{1}\left\|x_{n}\right\|_{p}^{p}-\int_{Z} v_{n}(z) x_{n}(z) d z \leq \varepsilon_{n}\left\|x_{n}\right\|
$$


Also, since $\left|\varphi\left(x_{n}\right)\right| \leq M_{1}$ for all $n \geq 1$, we have

$$
-\left\|D x_{n}\right\|_{p}^{p}+\lambda_{1}\left\|x_{n}\right\|_{p}^{p}+\int_{Z} p j\left(z, x_{n}(z)\right) d z \leq p M_{1} .
$$

Adding (3.11) and (3.12), we obtain

$$
\begin{aligned}
& \int_{Z}\left(p j\left(z, x_{n}(z)\right)-u_{n}(z) x_{n}(z)\right) d z \leq \varepsilon_{n}\left\|x_{n}\right\|+p M_{1} \\
& \Longrightarrow \int_{Z}\left(\frac{p j\left(z, x_{n}(z)\right)}{\left\|x_{n}\right\|}-u_{n}(z) v_{n}(z)\right) d z \leq \varepsilon_{n}+\frac{p M_{1}}{\left\|x_{n}\right\|} .
\end{aligned}
$$

Set

$$
h_{n}(z)= \begin{cases}\frac{j\left(z, x_{n}(z)\right)}{x_{n}(z)} & \text { if } x_{n}(z) \neq 0 \\ 0 & \text { if } x_{n}(z)=0 .\end{cases}
$$

Then we have

$$
\begin{aligned}
\varepsilon_{n}+\frac{p M_{1}}{\left\|x_{n}\right\|} & \geq \int_{Z} \frac{p j\left(z, x_{n}(z)\right)}{\left\|x_{n}\right\|} d z-\int_{Z} u_{n}(z) v_{n}(z) d z \\
& \geq \int_{Z} p h_{n}(z) v_{n}(z) d z-\int_{\left\{v_{n}>0\right\}} g_{1}\left(z, x_{n}(z)\right) v_{n}(z) d z \\
& -\int_{\left\{v_{n}<0\right\}} g_{0}\left(z, x_{n}(z)\right) v_{n}(z) d z \quad(\text { since } j(z, 0)=0 \text { a.e. on } Z) \\
& \geq \int_{\left\{v_{n}>0\right\}} G_{1}\left(z, x_{n}(z)\right) v_{n}(z) d z+\int_{\left\{v_{n}<0\right\}} \theta(z) v_{n}(z) d z
\end{aligned}
$$

(see hypothesis $\mathrm{H}(\mathrm{j})(\mathrm{v})$ and recall that $v_{n}(z) \geq 0$ a.e. on $Z$ ).

Recall that $v=u_{1}$ and $u_{1}(z)>0$ for all $z \in Z$. It follows that $x_{n}(z) \rightarrow+\infty$ a.e. on $Z$ as $n \rightarrow \infty$, and if by $\chi_{E}$ we denote the characteristic function of a measurable set $E \subseteq Z$, we have

$$
\chi_{\left\{v_{n}>0\right\}}(z) \longrightarrow 1, \quad \chi_{\left\{v_{n}<0\right\}}(z) \longrightarrow 0 \quad \text { a.e. on } Z \text {. }
$$

Also, from the definition of the function $G_{+}(\cdot)$ (see hypothesis $H(j)(v)$ ), given $\varepsilon>0$, we can find $M_{2}>0$ such that for almost all $z \in Z$ and all $x>M_{2}$, we have

$$
G_{1}(z, x) \geq G_{+}(z)-\varepsilon
$$

On the other hand, for almost all $z \in Z$ and all $x \in\left[0, M_{2}\right]$, we have

$$
G_{1}(z, x) \geq-\beta_{M_{2}}(z) \quad \text { with } \beta_{M_{2}} \in L^{1}(Z)_{+} .
$$

Therefore, for almost all $z \in Z$ and all $x \in \mathbb{R}_{+}$, we have

$$
G_{1}(z, x) \geq-\xi(z) \quad \text { with } \xi \in L^{1}(Z)_{+} .
$$


Passing to the limit as $n \rightarrow \infty$ in (3.15) and using Fatou's lemma (remark that (3.19) permits the use of Fatou's lemma), we obtain

$$
\int_{Z} G_{+}(z) u_{1}(z) d z \leq 0
$$

which contradicts hypothesis $\mathrm{H}(\mathrm{j})(\mathrm{v})$. Therefore, it follows that $\left\{x_{n}\right\}_{n \geq 1} \subseteq C$ is bounded in $W_{0}^{1, p}(Z)$, and so we may assume that $x_{n} \stackrel{w}{\rightarrow} x$ in $W_{0}^{1, p}(Z)$ and $x_{n} \rightarrow x$ in $L^{p}(Z)$, with $x \in C$. We have

$$
\begin{gathered}
\left\langle A\left(x_{n}\right), x_{n}-y\right\rangle-\lambda_{1} \int_{Z}\left|x_{n}\right|^{p-2} x_{n}\left(x_{n}-y\right) d z-\int_{Z} u_{n}\left(x_{n}-y\right) d z \\
\leq \varepsilon_{n}\left\|y-x_{n}\right\| \quad \forall y \in C .
\end{gathered}
$$

We fix $y=x \in C$. Hence we have

$$
\left\langle A\left(x_{n}\right), x_{n}-x\right\rangle-\lambda_{1} \int_{Z}\left|x_{n}\right|^{p-2} x_{n}\left(x_{n}-x\right) d z-\int_{Z} u_{n}\left(x_{n}-x\right) d z \leq \varepsilon_{n}\left\|x-x_{n}\right\| .
$$

Since $x_{n} \rightarrow x$ in $L^{p}(Z)$, we have $\int_{Z}\left|x_{n}\right|^{p-2} x_{n}\left(x_{n}-x\right) d z, \int_{Z} u_{n}\left(x_{n}-x\right) d z \rightarrow 0$ as $n \rightarrow \infty$. Hence,

$$
\limsup _{n \rightarrow \infty}\left\langle A\left(x_{n}\right), x_{n}-x\right\rangle \leq 0 \text {. }
$$

Recall that $A$ is maximal monotone, thus generalized pseudomonotone (see Denkowski et al. [5, page 37]). So we have

$$
\left\|D x_{n}\right\|_{p}^{p}=\left\langle A\left(x_{n}\right), x_{n}\right\rangle \longrightarrow\langle A(x), x\rangle=\|D x\|_{p}^{p} .
$$

Because $D x_{n} \stackrel{w}{\rightarrow} D x$ in $L^{p}\left(Z, \mathbb{R}^{N}\right)$ and $L^{p}\left(Z, \mathbb{R}^{N}\right)$ is uniformly convex, from the KadecKlee property, we have $D x_{n} \rightarrow D x$ in $L^{p}\left(Z, \mathbb{R}^{N}\right)$, hence $x_{n} \rightarrow x$ in $W_{0}^{1, p}(Z)$.

Proposition 3.3. If hypotheses $H(j)$ hold, then $\varphi\left(t u_{1}\right) \rightarrow-\infty$ as $t \rightarrow+\infty$.

Proof. Note that for all $t \geq 0, t u_{1} \in C$, and so

$$
\varphi\left(t u_{1}\right)=-\int_{Z} j\left(z, t u_{1}(z)\right) d z
$$

Recall that given $\varepsilon>0$, we can find $M_{2}=M_{2}(\varepsilon)>0$ such that for all $z \in Z \backslash D,|D|_{N}=0$ (by $|\cdot|_{N}$ we denote the Lebesgue measure on $R^{N}$ ), and all $x>M_{2}$, we have

$$
\begin{aligned}
& k_{\varepsilon}^{+}(z)=G_{+}(z)-\varepsilon \leq G_{1}(z, x) \\
& \Longrightarrow \frac{G_{1}(z, x)}{x^{p}} \geq \frac{k_{\varepsilon}^{+}(z)}{x^{p}}=\frac{d}{d x}\left(-\frac{1}{p-1} \frac{k_{\varepsilon}^{+}(z)}{x^{p-1}}\right) .
\end{aligned}
$$

For all $z \in Z \backslash D$, all $x>M_{2}$, and all $u \in \partial j(z, x)$, we have

$$
\frac{G_{1}(z, x)}{x^{p}}=\frac{p j(z, x)}{x^{p+1}}-\frac{g_{1}(z, x)}{x^{p}} \leq \frac{p j(z, x)}{x^{p+1}}-\frac{u}{x^{p}} .
$$


For all $z \in Z \backslash D$, the function $x \rightarrow p j(z, x) / x^{p}$ is locally Lipschitz on $\left(M_{2},+\infty\right)$ and

$$
\begin{aligned}
& \partial\left(\frac{j(z, x)}{x^{p}}\right) \subseteq \frac{\partial j(z, x) x^{p}-p j(z, x) x^{p-1}}{x^{2 p}}=\frac{\partial j(z, x)}{x^{p}}-\frac{p j(z, x)}{x^{p+1}} \\
& \Rightarrow \max \left[u^{\prime}: u^{\prime} \in \partial\left(\frac{j(z, x)}{x^{p}}\right)\right] \leq \frac{g_{1}(z, x)}{x^{p}}-\frac{p j(z, x)}{x^{p+1}}=-\frac{1}{x^{p}} G_{1}(z, x)
\end{aligned}
$$

(see Clarke [4, page 48] and Denkowski et al. [6, page 612]). So, for all $z \in Z \backslash D,|D|_{N}=0$, all $x>M_{2}$, and all $u^{\prime} \in \partial\left(j(z, x) / x^{p}\right)$, we have

$$
u^{\prime} \leq \frac{d}{d x}\left(\frac{1}{p-1} \frac{k_{\varepsilon}^{+}(z)}{x^{p-1}}\right)
$$

$(\operatorname{see}(3.26))$.

For fixed $z \in Z \backslash D,|D|_{N}=0$, the function $x \rightarrow j(z, x) / x^{p}$ is locally Lipschitz on $\left(M_{2},+\infty\right)$, hence it is differentiable at all $x \in\left(M_{2},+\infty\right) \backslash E_{1}(z)$, with $\left|E_{1}(z)\right|_{1}=0$. We set

$$
\begin{aligned}
& u_{0}^{\prime}(z, x)= \begin{cases}\frac{d}{d x}\left(\frac{j(z, x)}{x^{p}}\right) & \text { if } x \in\left(M_{2},+\infty\right) \backslash E(z), \\
0 & \text { otherwise, }\end{cases} \\
& \Longrightarrow u_{0}^{\prime}(z, x) \leq \frac{d}{d x}\left(\frac{1}{p-1} \frac{k_{\varepsilon}^{+}(z)}{x^{p-1}}\right) \quad \text { for almost all } x \in\left(M_{2},+\infty\right) .
\end{aligned}
$$

Consider $w, v>M_{2}, v>w$, and integrate the last inequality on the interval $[w, v]$. We obtain

$$
\frac{j(z, v)}{v^{p}}-\frac{j(z, w)}{w^{p}} \leq \frac{k_{\varepsilon}^{+}(z)}{p-1}\left(\frac{1}{v^{p-1}}-\frac{1}{w^{p-1}}\right) .
$$

We have seen in the proof of Proposition 3.2 that given $\varepsilon>0$, we can find $\beta_{\varepsilon} \in L^{1}(Z)_{+}$ such that for all $z \in Z \backslash D,|D|_{N}=0$, and all $x \in R$, we have

$$
\begin{aligned}
& |j(z, x)| \leq \beta_{\varepsilon}(z)+2 \varepsilon|x|^{p} \\
& \Longrightarrow \lim _{x \rightarrow+\infty} \frac{j(z, x)}{x^{p}}=0 \quad(\text { since } \varepsilon>0 \text { was arbitrary }) .
\end{aligned}
$$

So, returning to inequality (3.31) and passing to the limit as $v \rightarrow+\infty$, we obtain

$$
\begin{aligned}
& \frac{j(z, w)}{w^{p}} \geq \frac{k_{\varepsilon}^{+}(z)}{p-1} \frac{1}{w^{p-1}} \\
& \Longrightarrow \frac{j(z, w)}{w} \geq \frac{k_{\varepsilon}^{+}(z)}{p-1} \\
& \Longrightarrow \liminf _{w \rightarrow+\infty} \frac{j(z, w)}{w} \geq \frac{1}{p-1} G_{+}(z) \quad \text { (since } \varepsilon>0 \text { was arbitrary). }
\end{aligned}
$$


Suppose that the conclusion of the proposition was not true. This means that we can find $M_{3}>0$ and a sequence $\left\{t_{n}\right\}_{n \geq 1} \subseteq \mathbb{R}_{+}$with $t_{n} \rightarrow+\infty$, such that

$$
\begin{aligned}
& \varphi\left(t_{n} u_{1}\right)=-\int_{Z} j\left(z, t_{n} u_{1}(z)\right) d z \geq-M_{3}, \quad \forall n \geq 1, \\
& \Longrightarrow \frac{\varphi\left(t_{n} u_{1}\right)}{t_{n}}=-\int_{Z} \frac{j\left(z, t_{n} u_{1}(z)\right)}{t_{n} u_{1}(z)} u_{1}(z) d z \geq \frac{-M_{3}}{t_{n}} \quad\left(\text { recall } u_{1}(z)>0, \forall z \in Z\right) .
\end{aligned}
$$

Via Fatou's lemma and using (3.33), we obtain

$$
\int_{Z} G_{+}(z) u_{1}(z) d z \leq 0
$$

a contradiction to hypothesis $\mathrm{H}(\mathrm{j})(\mathrm{v})$. So, $\varphi\left(t u_{1}\right) \rightarrow-\infty$ as $t \rightarrow+\infty$.

Proposition 3.4. If hypotheses $H(j)$ hold, then $\varphi(x) \geq \beta_{1}\|x\|^{p}-\beta_{2}\|x\|^{\eta}$ for some $\beta_{1}, \beta_{2}>0$,

$$
p<\eta<p^{*}= \begin{cases}\frac{N}{N-p} & \text { if } N>p, \\ +\infty & \text { if } N \leq p\end{cases}
$$

and all $x \in W_{0}^{1, p}(Z)_{+}$.

Proof. By virtue of hypothesis $\mathrm{H}(\mathrm{j})(\mathrm{vi})$, we can find $\delta>0$ and $M_{4}>0$ such that for almost all $z \in Z$ and all $x>M_{4}$, we have

$$
j(z, x) \leq-\frac{\delta}{p} x^{p}
$$

If we combine this with hypothesis $\mathrm{H}(\mathrm{j})$ (iii) and the mean value theorem of locally Lipschitz functions (see Clarke [4, page 41] and Denkowski et al. [6, page 609]), for almost all $z \in Z$, all $x>0$, and for $\eta \in\left(p, p^{*}\right]$, we have

$$
j(z, x) \leq-\frac{\delta}{p} x^{p}+\xi_{1} x^{\eta} \quad \text { for some } \xi_{1}>0 .
$$

So, for all $x \in W_{0}^{1, p}(Z)_{+}$(i.e., $x \in W_{0}^{1, P}(Z)$ and $x(z) \geq 0$ a.e. on $Z$ ), we have

$$
\begin{aligned}
\varphi(x) & =\frac{1}{p}\|D x\|_{p}^{p}-\frac{\lambda_{1}}{p}\|x\|_{p}^{p}-\int_{Z} j(z, x(z)) d z \\
& \geq \frac{1}{p}\|D x\|_{p}^{p}-\frac{\lambda_{1}}{p}\|x\|_{p}^{p}+\frac{\delta}{p}\|x\|_{p}^{p}-\xi_{1}\|x\|_{\eta}^{\eta}
\end{aligned}
$$

( $\operatorname{see}(3.38))$.

Using Poincaré's inequality and the fact that $W_{0}^{1, p}(Z)$ is embedded continuously in $L^{\eta}(Z)$ (recall $\left.\eta \leq p^{*}\right)$, we see that we can find $\beta_{1}>0$ and $\beta_{2}>0$ such that

$$
\varphi(x) \geq \beta_{1}\|x\|^{p}-\beta_{2}\|x\|^{\eta} .
$$


Propositions 3.3 and 3.4 imply that the energy functional $\varphi$ has the generalized nonsmooth mountain pass geometry. So, applying Theorem 2.1, we will be able to prove the following existence theorem for problem (1.1).

Theorem 3.5. If hypotheses $H(j)$ hold, then problem (1.1) has a nontrivial solution $x \in$ $W_{0}^{1, p}(Z)$.

Proof. By virtue of Proposition 3.4, if we choose $r>0$ small, then for all $x \in W_{0}^{1, p}(Z)$ with $\|x\|=r$, we have

$$
\varphi(x) \geq \xi_{2}>0
$$

On the other hand, because of Proposition 3.3, we can find $t>r$ such that $\varphi\left(t u_{1}\right) \leq$ $\varphi(0) \leq 0$. These facts in conjunction with Proposition 3.2 permit the use of Theorem 2.1, which gives $x \in C$ such that

$\varphi(x) \geq \xi_{2}>0 \geq \varphi(0) \quad($ i.e., $x \neq 0), \quad \varphi_{1}^{0}(x ; h)+\varphi_{2}(x+h)-\varphi_{2}(x) \geq 0, \quad \forall h \in W_{0}^{1, p}(Z)$.

Set $\psi_{1}(h)=\varphi_{1}^{0}(x ; h)$ and $\psi_{2}(h)=\varphi_{2}(x+h)-\varphi_{2}(x)$. Then $\psi_{1}: W_{0}^{1, p}(Z) \rightarrow \mathbb{R}$ is continuous, convex, while $\psi_{2} \in \Gamma_{0}\left(W_{0}^{1, p}(Z)\right)$. Remark that $\partial_{c} \psi_{1}(0)=\partial \varphi_{1}(x)$ and $\partial_{c} \psi_{2}(0)=$ $\partial_{c} \varphi_{2}(x)$. Moreover, from the second inequality in (3.42), we have

$$
\psi_{1}(h)+\psi_{2}(h) \geq 0 \Longrightarrow 0 \in \partial_{c}\left(\psi_{1}+\psi_{2}\right)(0) \quad\left(\text { since } \psi_{1}(0)=\psi_{2}(0)=0\right) .
$$

But from convex analysis (see Denkowski et al. [6, page 549]), we have that

$$
\partial_{c}\left(\psi_{1}+\psi_{2}\right)(0)=\partial_{c} \psi_{1}(0)+\partial_{c} \psi_{2}(0)=\partial \varphi_{1}(x)+\partial_{c} \varphi_{2}(x)
$$

We know that $\partial_{c} \varphi_{2}(x)=N_{C}(x)=$ the normal cone to $C$ at $x$ (see Denkowski et al. [6, page 622]). From (3.43) and (3.44), we see that we can find $x^{*} \in \partial \varphi_{1}(x)$ and $v^{*} \in$ $\partial_{c} \varphi_{2}(x)=N_{C}(x)$ such that $x^{*}+v^{*}=0$. We have

$$
x^{*}=A(x)-\lambda_{1}|x|^{p-2} x-u
$$

with $u \in L^{q}(Z), u(z) \in \partial j(z, x(z))$ a.e on $Z$, and $\left\langle v^{*}, x-y\right\rangle \geq 0$ for all $y \in C$.

It follows that $\left\langle x^{*}, y-x\right\rangle \geq 0$ for all $y \in C$. Therefore, we have

$$
\begin{aligned}
& \left\langle A(x)-\lambda_{1}|x|^{p-2} x-u, y-x\right\rangle \geq 0 \quad \forall y \in C, \\
& \Longrightarrow \int_{Z}\|D x\|^{p-2}(D x, D(y-x))_{R^{N}} d z-\lambda_{1} \int_{Z}|x|^{p-2} x(y-x) d z \\
& \quad \geq \int_{Z} u(y-x) d z \quad \forall y \in C, \\
& \Longrightarrow x \in W_{0}^{1, p}(Z) \text { is a nontrivial solution of problem }(1.1) .
\end{aligned}
$$




\section{Positive solutions}

In this section, we deal with problem (1.2). Having as our starting point the existence theorem of the previous section, we establish that problem (1.2) has a nontrivial positive solution.

Theorem 4.1. If hypotheses $H(j)$ hold, there exists $M_{0}>0$ such that for almost all $z \in Z$, all $x \geq M_{0}$, and all $u \in \partial j(z, x), u \geq 0$ or $u \leq 0$ and $\partial j(z, 0) \subseteq R_{+}$a.e. on $Z$, then problem (1.2) has a nontrivial solution $x \in W_{0}^{1, p}(Z)_{+}$(i.e., $x(z) \geq 0$ a.e. on $Z$ ).

Proof. Let $C=W_{0}^{1, p}(Z)_{+}=\left\{x \in W_{0}^{1, p}(Z): x(z) \geq 0\right.$ a.e. on $\left.Z\right\}$ (so the obstacle now is $g \equiv$ 0 ) and let $\varphi_{1}, \varphi_{2}$, and $\varphi$ be as before. From Theorem 3.5, we know that there exists $x \in C$, $x \neq 0$, such that $x$ is a critical point of $\varphi=\varphi_{1}+\varphi_{2}$. So we can find $x^{*} \in \partial \varphi_{1}(x)$ such that

$$
x^{*}=A(x)-\lambda_{1}|x|^{p-2} x-u, \quad \text { with } u \in L^{q}(Z), u(z) \in \partial j(z, x(z)) \text { a.e. on } Z,
$$

and $\left\langle x^{*}, y-x\right\rangle \geq 0$ for all $y \in C$.

First we assume that for almost all $z \in Z$, all $x \geq M_{0}$, and all $u \in \partial j(z, x)$, we have $u \geq 0$.

Let $\theta \in W_{0}^{1, p}(Z), \varepsilon>0$, and $y=(x+\varepsilon \theta)^{+}=x+\varepsilon \theta+(x+\varepsilon \theta)^{-}$. We obtain

$$
\begin{aligned}
\left\langle x^{*}, \varepsilon \theta\right\rangle & \geq-\left\langle x^{*},(x+\varepsilon \theta)^{-}\right\rangle \\
& =-\left\langle A(x),(x+\varepsilon \theta)^{-}\right\rangle+\lambda_{1} \int_{Z}|x|^{p-2} x(x+\varepsilon \theta)^{-} d z+\int_{Z} u(x+\varepsilon \theta)^{-} d z .
\end{aligned}
$$

Set $Z_{-}^{\varepsilon}=\{z \in Z:(x+\varepsilon \theta)(z)<0\}$. We have

$$
\begin{aligned}
& -\left\langle A(x),(x+\varepsilon \theta)^{-}\right\rangle \\
& =-\int_{Z}\|D x\|^{p-2}\left(D x, D(x+\varepsilon \theta)^{-}\right)_{R^{N}} d z \\
& =\int_{Z_{-}^{\varepsilon}}\|D x\|^{p-2}(D x, D(x+\varepsilon \theta))_{R^{N}} d z \\
& \geq \varepsilon \int_{Z_{-}^{\varepsilon}}\|D x\|^{p-2}(D x, D \theta)_{R^{N}} d z
\end{aligned}
$$

(see [6, page 348]).

Also, we have

$$
\begin{aligned}
& \lambda_{1} \int_{Z}|x|^{p-2} x(x+\varepsilon \theta)^{-} d z \\
& \quad=-\lambda_{1} \int_{Z_{-}^{\varepsilon}}|x|^{p-2} x(x+\varepsilon \theta) d z \geq 0 \quad\left(\text { since } x \in C \text { and }\left.(x+\varepsilon \theta)\right|_{Z_{-}^{\varepsilon}}<0\right) .
\end{aligned}
$$

In addition, we have

$$
\begin{aligned}
\int_{Z} u(x+\varepsilon \theta)^{-} d z & =-\int_{Z_{-}^{\varepsilon}} u(x+\varepsilon \theta) d z \\
& =-\int_{Z_{-}^{\varepsilon} \cap\left\{x<M_{0}\right\}} u(x+\varepsilon \theta) d z-\int_{Z_{-}^{\varepsilon} \cap\left\{x \geq M_{0}\right\}} u(x+\varepsilon \theta) d z .
\end{aligned}
$$


By hypothesis, we have

$$
-\int_{Z_{-}^{\varepsilon} \cap\left\{x \geq M_{0}\right\}} u(x+\varepsilon \theta) d z \geq 0 .
$$

Also, since by hypothesis, $\partial j(z, 0) \subseteq R_{+}$a.e. on $Z$, we see that $u(z) \geq 0$ a.e. on $Z_{-}^{\varepsilon} \cap\{x=$ 0 \}. Moreover, remark that since $x(z) \geq 0$ a.e. on $Z$, we have $\theta(z)<0$ a.e. on $Z_{-}^{\varepsilon}$. So we obtain

$$
\begin{aligned}
& -\int_{Z_{-}^{\varepsilon} \cap\{x=0\}} u \varepsilon \theta d z \geq 0 \\
& \Longrightarrow-\int_{Z_{-}^{\varepsilon} \cap\left\{x<M_{0}\right\}} u(x+\varepsilon \theta) d z \\
& \quad \geq-\int_{Z_{-}^{\varepsilon} \cap\left\{0<x<M_{0}\right\}} u(x+\varepsilon \theta) d z \\
& \quad \geq \xi_{3} \int_{Z_{-}^{\varepsilon} \cap\left\{0<x<M_{0}\right\}}(x+\varepsilon \theta) d z \quad \text { for some } \xi_{3}>0 \\
& \quad \geq \xi_{3} \varepsilon \int_{Z_{-}^{\varepsilon} \cap\left\{0<x<M_{0}\right\}} \theta d z \quad(\text { since } x(z) \geq 0 \text { a.e. on } Z)
\end{aligned}
$$

(see hypothesis $H(j)($ iii)).

Using (4.6) and (4.7) in (4.5), we have

$$
\int_{Z} u(x+\varepsilon \theta)^{-} d z \geq \xi_{3} \varepsilon \int_{Z_{-}^{\varepsilon} \cap\left\{0<x<M_{0}\right\}} \theta d z
$$

We return to (4.2) and we use (4.3), (4.4), and (4.8). We obtain

$$
\left\langle x^{*}, \theta\right\rangle \geq \int_{Z_{-}^{\varepsilon}}\|D x\|^{p-2}(D x, D \theta)_{R^{N}} d z+\xi_{3} \int_{Z_{-}^{\varepsilon} \cap\left\{0<x<M_{0}\right\}} \theta d z .
$$

Recall that $D x(z)=0$ a.e. on $\{x=0\}$ (see Denkowski et al. [6, page 349]). Also note that $\left|Z_{-}^{\varepsilon} \cap\left\{0<x<M_{0}\right\}\right|_{N} \rightarrow 0$ as $\varepsilon \downarrow 0$ (by $|\cdot|_{N}$ we denote the Lebesgue measure on $\mathbb{R}^{N}$ ). So, if we pass to the limit as $\varepsilon \downarrow 0$, we obtain

$$
\begin{aligned}
& \left\langle x^{*}, \theta\right\rangle \geq 0, \quad \forall \theta \in W_{0}^{1, p}(Z) \\
& \Longrightarrow x^{*}=A(x)-\lambda_{1}|x|^{p-2} x-u=0 \\
& -\operatorname{div}\left(\| D x(z)||^{p-2} D x(z)\right)-\lambda_{1}|x(z)|^{p-2} x(z)=u(z) \quad \text { a.e on } Z,\left.x\right|_{\partial Z}=0 \\
& \Longrightarrow x \in W_{0}^{1, P}(Z)_{+} \text {is a nontrivial solution of }(1.2)
\end{aligned}
$$

Next assume that for almost all $z \in Z$, all $x \geq M_{0}$, and all $u \in \partial j(z, x)$, we have $u \leq 0$. Then we have (see hypothesis $\mathrm{H}(\mathrm{j})$ (iii) and recall that $\partial j(z, 0) \subseteq R_{+}$a.e. on $Z$ )

$$
-\int_{Z_{-}^{\varepsilon} \cap\left\{x<M_{0}\right\}} u(x+\varepsilon \theta) d z \geq \xi_{3} \varepsilon \int_{Z_{-}^{\varepsilon} \cap\left\{0<x<M_{0}\right\}} \theta d z .
$$


Also we have

$$
\left.-\int_{Z_{-}^{\varepsilon} \cap\left\{x \geq M_{0}\right\}} u(x+\varepsilon \theta) d z \geq-\varepsilon \int_{Z_{-}^{\varepsilon} \cap\left\{x \geq M_{0}\right\}} u \theta d z \quad \text { (since }-u x \geq 0\right) .
$$

Remark that $\left|Z_{-}^{\varepsilon} \cap\left\{x \geq M_{0}\right\}\right|_{N} \rightarrow 0$ as $\varepsilon \downarrow 0$. Using (4.3), (4.4), (4.11), and (4.12) in (4.2), we obtain

$$
\begin{aligned}
& \left\langle x^{*}, \theta\right\rangle \geq \int_{Z_{-}^{\varepsilon}}\|D x\|^{p-2}(D x, D \theta)_{R^{N}} d z+\xi_{3} \int_{Z_{-}^{\varepsilon} \cap\left\{0<x<M_{0}\right\}} \theta d z-\int_{Z_{-}^{\varepsilon} \cap\left\{x \geq M_{0}\right\}} u \theta d z \\
& \left.\Longrightarrow\left\langle x^{*}, \theta\right\rangle \geq 0 \quad \forall \theta \in W_{0}^{1, p}(Z) \text { (by passing to the limit as } \varepsilon \downarrow 0\right) \\
& \Longrightarrow x^{*}=A(x)-\lambda_{1}|x|^{p-2} x-u=0 \\
& \Longrightarrow x=\text { solution of }(1.2) .
\end{aligned}
$$

If we strengthen our hypotheses on $j$, we can have that $x$ is smooth and strictly positive.

Theorem 4.2. If hypotheses $H(j)$ hold, there exists $M_{0}>0$ such that for almost all $z \in Z$, all $x \geq M_{0}$, and all $u \in \partial j(z, x), u \geq 0$ or $u \leq 0, \partial j(z, 0) \subseteq \mathbb{R}_{+}$a.e. on $Z$, and for almost all $z \in Z$, all $x \in \mathbb{R}_{+}$, and all $u \in \partial j(z, x), u \geq-\xi_{4} x^{p-1}$ for some $\xi_{4} \geq \lambda_{1}$, then problem (1.2) has a solution $x \in C^{1}(\bar{Z})$ such that $x(z)>0$ for all $z \in Z$ and $\partial x / \partial n<0$ on $\partial Z$.

Proof. Let $x \in W_{0}^{1, p}(Z)_{+}$be the nontrivial solution of (1.2) obtained in Theorem 4.1. From Ladyzhenskaya and Ural'tseva [12, page 286], we know that $x \in L^{\infty}(Z)_{+}$. Invoking [15, Theorem 1], we infer that $x \in C^{1}(\bar{Z})_{+}$.

Moreover, since $-\operatorname{div}\left(\|D x(z)\|^{p-2} D x(z)\right)=u(z)+\lambda_{1}|x(z)|^{p-2} x(z) \geq\left(-\xi_{4}+\lambda_{1}\right)|x(z)|^{p-1}$ a.e. on $Z$, we can apply [20, Theorem 5] to conclude that $x(z)>0$ for all $z \in Z$ and $\partial x / \partial n<0$ on $\partial Z$.

Remark 4.3. If, for simplicity, we drop the $z$-dependence, the following function satisfies the hypotheses in all the theorems of this paper:

$$
j(x)= \begin{cases}\ln \left(1+x^{2}\right) & \text { if } x<0, \\ -x^{r} & \text { if } x \in[0,1], \text { with } r<p, \\ x-2 & \text { if } x \geq 1 .\end{cases}
$$

\section{Acknowledgment}

The first author was supported by a grant of the National Scholarship Foundation of Greece (IKY).

\section{References}

[1] C. O. Alves and O. H. Miyagaki, Existence of positive solutions to a superlinear elliptic problem, Electron. J. Differential Equations 2001 (2001), no. 11, 1-12.

[2] K. C. Chang, Variational methods for nondifferentiable functionals and their applications to partial differential equations, J. Math. Anal. Appl. 80 (1981), no. 1, 102-129.

[3] R. Chiappinelli, J. Mawhin, and R. Nugari, Bifurcation from infinity and multiple solutions for some Dirichlet problems with unbounded nonlinearities, Nonlinear Anal. 18 (1992), no. 12, 1099-1112. 
[4] F. H. Clarke, Optimization and Nonsmooth Analysis, Canadian Mathematical Society Series of Monographs and Advanced Texts, John Wiley \& Sons, New York, 1983.

[5] Z. Denkowski, S. Migórski, and N. S. Papageorgiou, An Introduction to Nonlinear Analysis. Applications, Kluwer Academic/Plenum Publishers, New York, 2003.

[6] _ An Introduction to Nonlinear Analysis. Theory, Kluwer Academic/Plenum Publishers, New York, 2003.

[7] D. Goeleven, D. Motreanu, and P. D. Panagiotopoulos, Eigenvalue problems for variationalhemivariational inequalities at resonance, Nonlinear Anal. 33 (1998), no. 2, 161-180.

[8] S. Hu and N. S. Papageorgiou, Handbook of Multivalued Analysis. Vol. II. Applications., Mathematics and Its Applications, vol. 500, Kluwer Academic Publishers, Dordrecht, 2000.

[9] N. C. Kourogenis, J. Papadrianos, and N. S. Papageorgiou, Extensions of nonsmooth critical point theory and applications, Atti Sem. Mat. Fis. Univ. Modena 50 (2002), no. 2, 381-414.

[10] N. C. Kourogenis and N. S. Papageorgiou, Nonsmooth critical point theory and nonlinear elliptic equations at resonance, J. Austral. Math. Soc. Ser. A 69 (2000), no. 2, 245-271.

[11] S. Kyritsi and N. S. Papageorgiou, Multiple solutions of constant sign for nonlinear nonsmooth eigenvalue problems near resonance, Calc. Var. Partial Differential Equations 20 (2004), no. 1, $1-24$.

[12] O. A. Ladyzhenskaya and N. N. Ural'tseva, Linear and Quasilinear Elliptic Equations, Academic Press, New York, 1968.

[13] V. K. Le, Existence of positive solutions of variational inequalities by a subsolution-supersolution approach, J. Math. Anal. Appl. 252 (2000), no. 1, 65-90.

[14] Subsolution-supersolution method in variational inequalities, Nonlinear Anal. 45 (2001), no. 6, 775-800.

[15] G. M. Lieberman, Boundary regularity for solutions of degenerate elliptic equations, Nonlinear Anal. 12 (1988), no. 11, 1203-1219.

[16] J. Mawhin and K. Schmitt, Nonlinear eigenvalue problems with the parameter near resonance, Ann. Polon. Math. 51 (1990), 241-248.

[17] Z. Naniewicz and P. D. Panagiotopoulos, Mathematical Theory of Hemivariational Inequalities and Applications, Monographs and Textbooks in Pure and Applied Mathematics, vol. 188, Marcel Dekker, New York, 1995.

[18] M. Struwe, Variational Methods, Springer-Verlag, Berlin, 1990.

[19] A. Szulkin, Minimax principles for lower semicontinuous functions and applications to nonlinear boundary value problems, Ann. Inst. H. Poincaré Anal. Non Linéaire 3 (1986), no. 2, 77109.

[20] J. L. Vázquez, A strong maximum principle for some quasilinear elliptic equations, Appl. Math. Optim. 12 (1984), no. 3, 191-202.

[21] H.-S. Zhou, Existence of asymptotically linear Dirichlet problem, Nonlinear Anal. 44 (2001), no. 7, 909-918.

[22] Y. Zhou and Y. Huang, Existence of solutions for a class of elliptic variational inequalities, J. Math. Anal. Appl. 250 (2000), no. 1, 187-195.

Michael E. Filippakis: Department of Mathematics, National Technical University of Athens, Zografou Campus, Athens 15780, Greece

Nikolaos S. Papageorgiou: Department of Mathematics, National Technical University of Athens, Zografou Campus, Athens 15780, Greece

E-mail address: npapg@math.ntua.gr 


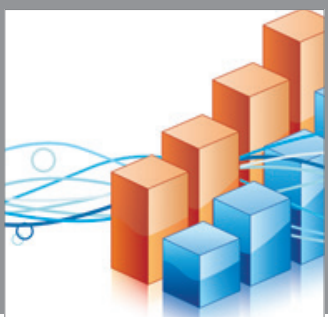

Advances in

Operations Research

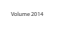

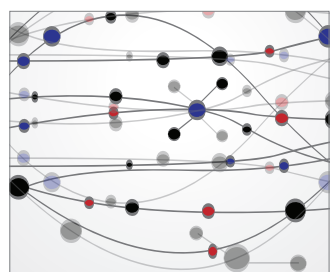

\section{The Scientific} World Journal
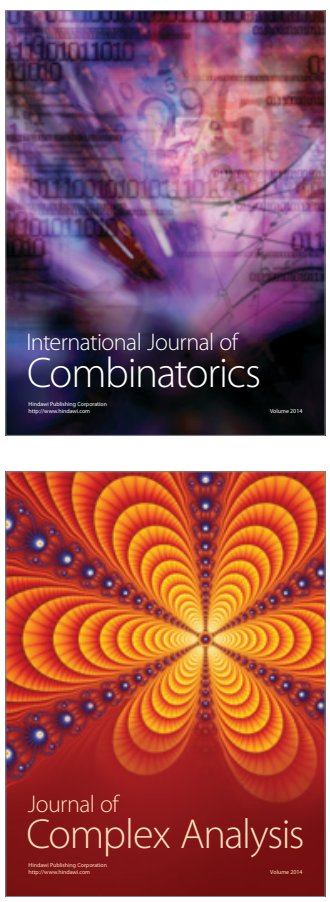

International Journal of

Mathematics and

Mathematical

Sciences
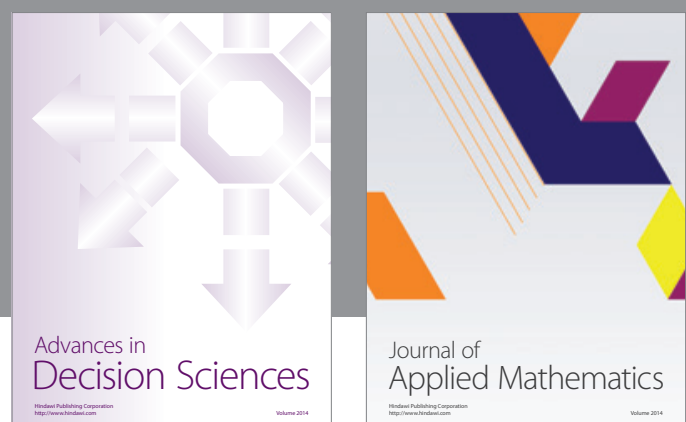

Journal of

Applied Mathematics
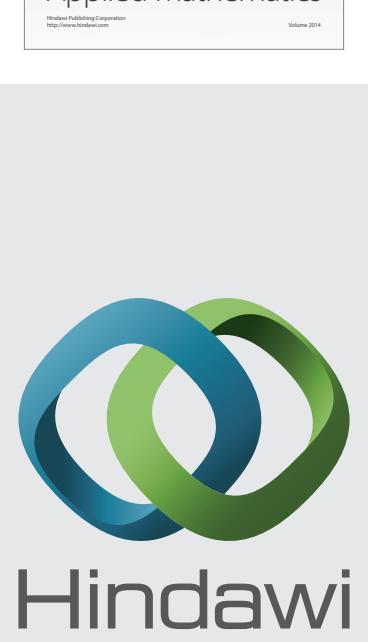

Submit your manuscripts at http://www.hindawi.com
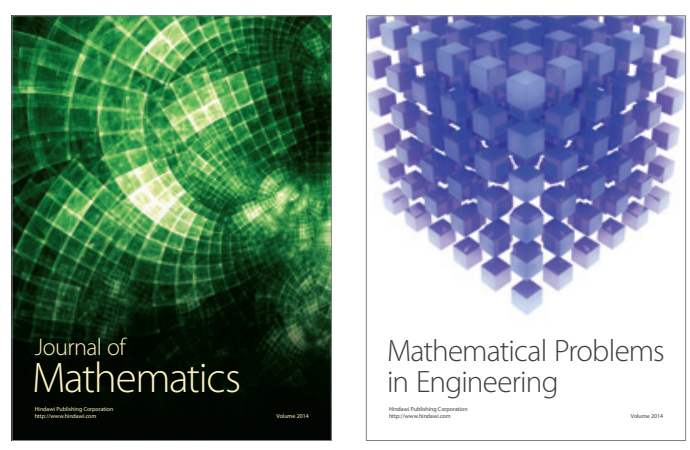

Mathematical Problems in Engineering
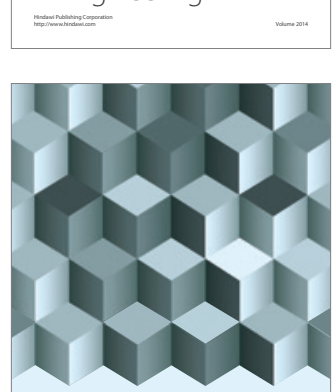

Journal of

Function Spaces
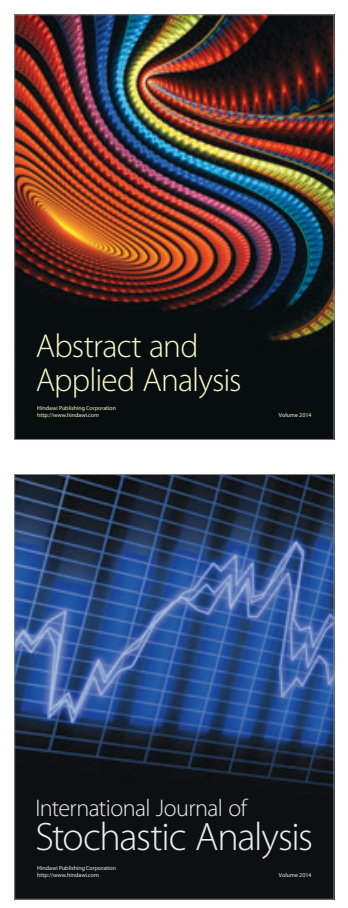

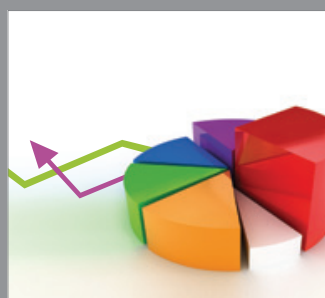

ournal of

Probability and Statistics

Promensencen
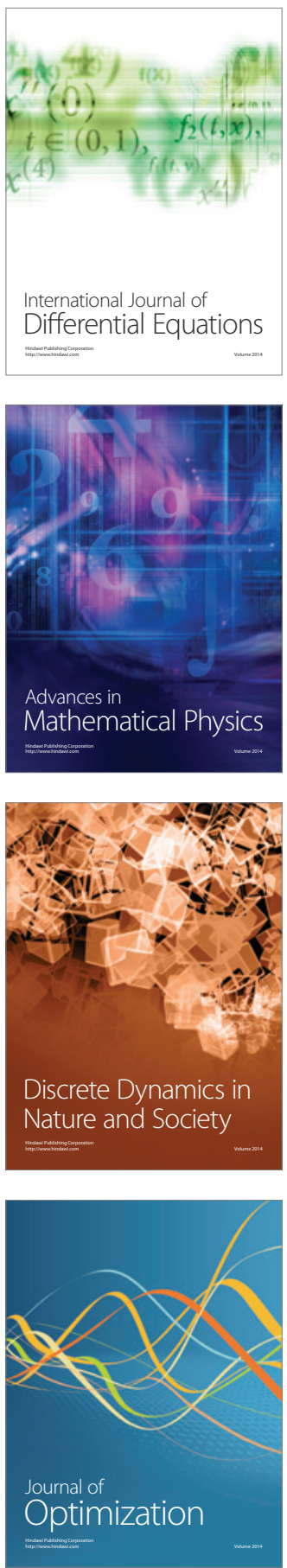